\title{
The Effect of T'ai Chi Exercise on Immunity and Infections: A Systematic Review of Controlled Trials
}

\author{
Rainbow T.H. Ho, PhD, ${ }^{1,2}$ Chong-Wen Wang, MD, PhD, Siu-Man Ng, PhD, 2,3 Andy H.Y. Ho, MSocSc, \\ Eric T.C. Ziea, MD, PhD, ${ }^{4}$ Vivian Taam Wong, MD, FRCP, ${ }^{4}$ and Cecilia L.W. Chan, $\mathrm{PhD}^{1,2}$
}

\begin{abstract}
Purpose: The aim of this review is to summarize and assess critically clinical trial evidence of the effect of $t^{\prime} a i$ chi (TC) exercise on immunity and TC efficacy for treating infectious diseases.

Methods: Fourteen databases were searched from their respective inceptions through January 2011. No language restrictions were imposed. Quality and validity of the included clinical trials were evaluated using standard scales.

Results: Sixteen (16) studies, including 7 randomized controlled trials, 4 controlled clinical trials, and 5 retrospective case-control studies, met the inclusion criteria for this review. One (1) study examined clinical symptoms, 3 studies tested functional measures of immunity (antigen-induced immunity), and the other studies tested enumerative parameters of immunity. such as lymphocytes, immunoglobulins, complements, natural-killer cells, and myeloid dendritic cells. Overall, these studies suggested favorable effects of TC exercise.

Conclusions: TC exercise appears to improve both cell-mediated immunity and antibody response in immune system, but it remains debatable whether or not the changes in immune parameters are sufficient to provide protection from infections.
\end{abstract}

\section{Introduction}

$T$ 'AI Chi (TC), A COMPlementary AND ALternative MODALITY of Traditional Chinese Medicine, combines characteristics of physical exercise and meditative practice. TC is popularly practiced by a large number of people in Chinese communities to improve physical fitness and overall well-being. The gentle movements and postures of the exercise coordinated with breathing patterns and meditation are designed to achieve a harmonious flow of energy (qi) in the body. TC exercise is equivalent to moderate-intensity aerobic exercise. However, TC is not only an exercise but also a mindbody intervention. Its beneficial effects on health have been documented in increasing number of studies. ${ }^{1-3}$ It is hypothesized that TC as a modality of mind-body intervention with a moderate intensity of physical exercise may improve immune functions of human body. ${ }^{4,5}$ However, few reviews have examined the scientific evidence of the effect of TC on immunity. It is well-known that infections, such as influenza and herpes zoster, are associated with human body's immunity, and that individuals who have substantial declines in immune function are at increased risk for contracting a number of infectious diseases. Epidemiologic studies have suggested that moderate exercise training is associated with reduction in the incidence of upper respiratory-tract infection (URTI), whereas endurance athletes are at increased risk for URTIs during periods of heavy training. ${ }^{5,6}$ It is still unclear whether or not TC exercise may reduce the incidence or the severity of infectious diseases. Thus, this systematic review aims to summarize and evaluate critically clinical trial evidence of the effectiveness of TC exercise for improving immune functions and its efficacy for treating infectious diseases.

\section{Methods}

\section{Data sources}

The following electronic databases were searched from their respective inceptions through January 2011: PubMed/ MEDLINE; CENTRAL; CINAHL; EMBASE; AMED; Qigong and Energy Medicine Database; SPORTDiscus Database; China Journals Full-text Database-Medicine/Hygiene Series, China Proceedings of Conference Full-text Database, Chinese Master Theses Full-text Database, China Doctor Dissertations

\footnotetext{
${ }^{1}$ Center on Behavioral Health, ${ }^{2}$ Department of Social Work and Social Administration, and ${ }^{3}$ Family Institute, The University of Hong Kong, Hong Kong SAR, China.

${ }^{4}$ Chinese Medicine Department, Hospital Authority of Hong Kong, Hong Kong SAR, China.
} 
Full-text Database, Electronic Theses and Dissertation System (Taiwan), Taiwan Electronic Periodical Services, and Index to Taiwan Periodical Literature System. The search terms used for this review included: tai chi, taichi, tai ji, taiji, taijichuan, shadowboxing, influenza, infection, infectious, inflammation, inflammatory, immune, immunity, immunological, lymphocyte, and antibody. Both traditional and simplified Chinese translations of these terms were used in Chinese databases. Reference lists of all included studies, existing reviews, and other archives of the located publications were hand-searched for further relevant articles.

\section{Study selection}

All controlled clinical trials (CCTs) were included if they examined the effects of TC exercise on the parameters of immunity or for treatment of various infectious diseases. Given the limited number of prospective clinical trials in the field, retrospective case-control studies (RCSs) were also included to provide alternative evidence, but uncontrolled observational studies were excluded, because of their susceptibility to bias and lack of significant evidence. Case reports and qualitative studies were also excluded for lack of significant evidence. To assess the effect of TC on immunity, studies on any subject were included, but studies among athletes were excluded because of their high intensity of physical exercise. To evaluate the effect of TC on infections, any study about TC concerning the incidence or severity of infectious diseases were included. For all included studies, primary data from the original sources were reviewed and analyzed.

To assess the effects of TC exercise on improvement of immunity and TC's effectiveness for treating infectious diseases, such outcome measures as physical symptoms relevant to infections and biomedical indicators of immunity were considered. Generally, an individual's immune status can be assessed using either enumerative or functional measures. ${ }^{7}$ Functional measures assess how well a specific immune process works (e.g., how effectively natural-killer [NK] cells destroy laboratory-grown tumor cells or how much lymphocytes divide following stimulation with a mitogen [a substance that induces mitosis or cell division]). In clinical practice, one of the most valid and commonly used functional measures of immunity is used to assess people's immune responses to antigens that people are highly sensitive to. This technique involves placing a small piece of antigen directly underneath the skin, a procedure that causes a local inflammatory response consisting of induration (a swollen round bump) and erythema (redness around the bump), and measuring the magnitude of this response immediately or $24-48$ hours later, depending on the antigen that is used. Enumerative measures involve counting different immune-system components (or biomarkers), including white blood-cell populations (granulocytes, monocytes, lymphocytes, NK cells, B-lymphocytes, T-lymphocytes, helper T-lymphocytes, and suppressor/cytotoxic T-lymphocytes), antibody populations in the blood (immunoglobulin [Ig]A, IgG, and IgM) and in saliva (secretory $\operatorname{IgA}$ ), and antibodies to specific pathogens. The current review focused on the number and the percentage of white blood cells, mainly T-lymphocytes, and levels of serum Igs and complements in peripheral blood, because these biomarkers are commonly used parameters in clinical practice and in the field of exer- cise research. Psychosocial outcomes, such as quality of life and psychologic well-being, were not considered because it is difficult to attribute effects on such outcomes to the change of immunity.

\section{Data extraction and assessment}

For each included study, data were extracted by 1 main researcher and then verified by another researcher. All discrepancies were resolved by discussion. The strength of the evidence was evaluated for all the included studies using the Oxford Centre for Evidence-based Medicine Levels of Evidence. ${ }^{8}$ These criteria are applied to grade the methodological rigor of studies from level 1 or grade A (systematic review of RCTs, 1a; individual RCT with narrow confidence interval, 1b) to level 5 or grade D (expert opinion). The quality and validity of the included RCTs were also evaluated using the Jadad scale, ${ }^{9}$ which is based on three criteria: (1) description of randomization and allocation concealment; (2) double-blinding; and (3) withdrawals or dropouts (the score ranges from 0 to 5). This is a standard scale used in systematic reviews of RCTs. Given that it was difficult to blind patients to TC, only assessor blinding was evaluated. The risk of bias in the included trials was assessed using the framework for methodological quality recommended by Juni et al. $^{10}$ According to this framework, biases fall into four categories: (1) selection bias (biased allocation to comparison groups); (2) performance bias (unequal provision of care apart from intervention under evaluation); (3) detection bias (biased assessment of outcomes); and (4) and attrition bias (biased occurrence of loss to follow-up).

\section{Results}

\section{Study description}

The database searches identified 87 potentially relevant articles (Fig. 1). Of them, 51 articles were excluded because they were not clinical trials or not related to infection or immunity. Full reports of 36 studies were acquired, and 20 were also excluded because they were uncontrolled observational studies (10), studies with unparallel controls (2), duplicate publications (3), studies using athletes (2), and studies with other outcome measures (3). Sixteen studies published between 1988 and 2010, including 7 RCTs, ${ }^{11-17} 4$ CCTs, $^{18-21}$ and $5 \mathrm{RCSS}^{22-26}$ met this review's inclusion criteria. These studies were conducted in the United States, ${ }^{13,14,16,19}$ Taiwan, ${ }^{22}$ and mainland China. ${ }^{11,12,15,17,18,20,21,23-26}$ Seven (7) studies $^{13,14,16,19,22,24,25}$ were published in English, 8 studies $^{11,12,15,17,18,21,23,26}$ were published in Chinese, and the remaining 1 study $^{20}$ was a proceeding.

Of the 16 included studies, 4 used samples of young college students; ${ }^{11,12,18,20} 1$ study used a sample of persons with HIV infection, ${ }^{16}$ and the other studies used samples of middle-age or older healthy adults. Four (4) studies $^{11,12,17,18}$ focused solely on females and 2 studies ${ }^{22,23}$ focused solely on males. Sample sizes in the included studies ranged from 16 to 252. A sample shared by 2 studies $^{11,12}$ was considered to be one sample. In total, these studies covered 939 subjects, including 577 subjects in the TC exercise groups and 362 subjects in the control groups. Characteristics of the included RCTs and non-RCTs (CCTs and RCSs) are presented in Tables 1 and 2, respectively. 


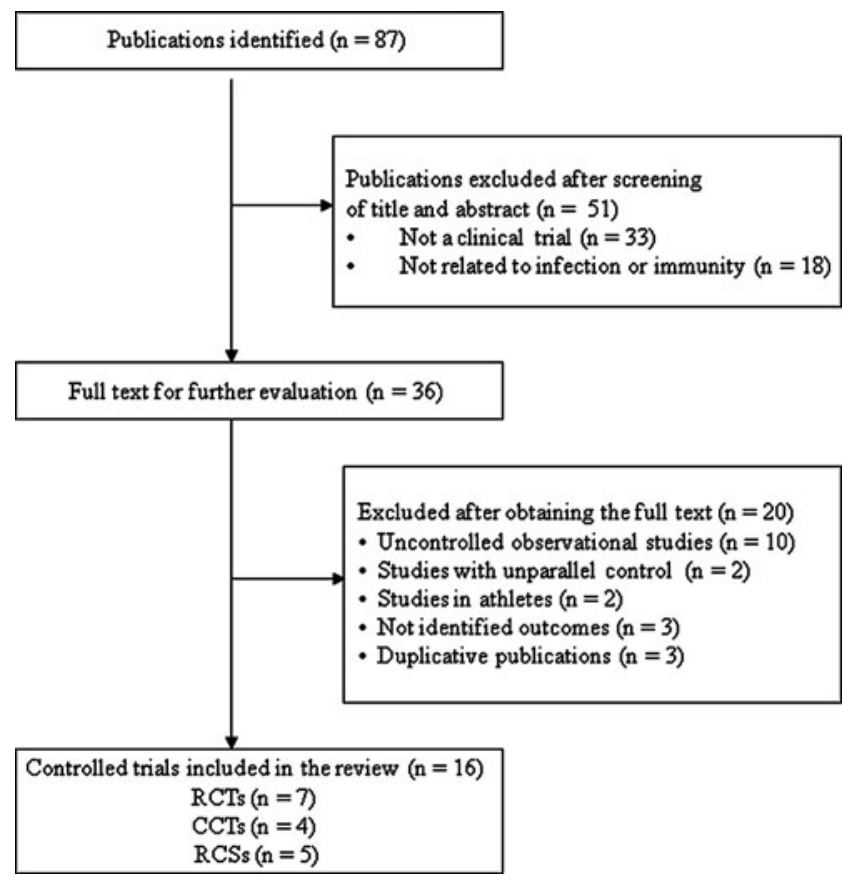

FIG. 1. Selection process for included studies. RCTs, randomized controlled trials; CCTs, clinical controlled trials; RCSs, retrospective case-control studies.

Durations of TC intervention ranged from 5 weeks to 6 months for the 11 prospective studies (7 RCTs and 4 CCTs). The five $\mathrm{RCSs}^{22-26}$ reported durations of TC practice spanning 12 months to 12 years. The majority of the included studies were conducted with a two-armed parallel-group design except for 2 studies ${ }^{18,20}$ with a 3 -armed parallel group-design and 3 studies $^{11,12,16}$ with a 4 -armed parallel group design. One (1) study ${ }^{20}$ compared TC with qigong, and another study ${ }^{13}$ used group health education as a control while the other studies used a wait-list group or a group with routine activites as a control.

According to outcome measures, these studies could be divided into 3 categories: (1) 1 study ${ }^{18}$ on clinical symptoms relevant to infections; (2) 3 studies ${ }^{13,14,19}$ on functional measures of immunity (antigen-induced immunity); and (3) other studies on enumerative parameters of immunity. The mostoften used outcome measure in the included studies was Tcells ${ }^{15,16,21,23-26}$ followed by $\operatorname{Igs}$ (IgA, IgE, IgG, and IgM), ${ }^{12,15,18,21}$ complements (C3, C4), ${ }^{11,20}$ NK-cells, ${ }^{17,23}$ and myeloid dendritic cells. ${ }^{22}$

\section{Effects of TC intervention}

One $\mathrm{CCT}^{18}$ on clinical symptoms suggested a favorable effect of TC exercise. Three (3) studies, including 2 RCTs $^{13,14}$ and $1 \mathrm{CCT}^{19}$ examined the effects of TC on antigen-induced, virus-specific cell-mediated immunity and antibody response in the human immune system. The results of these studies indicated that TC exercise could augment immune responses to virus and influenza vaccines.

Six (6) studies, including $2 \mathrm{RCTs},{ }^{15,16} 1 \mathrm{CCT}^{21}$ and 3 $\mathrm{RCSs}^{23,25,26}$ examined number and/or percentage of $\mathrm{T}$ lymphocytes. One (1) $\mathrm{RCT}^{15}$ on older adults suggested that the number of $\mathrm{CD} 4^{+}$and the ratio of $\mathrm{CD} 4^{+} / \mathrm{CD} 8^{+}$increased significantly after 8 weeks of TC practice. Another $\mathrm{RCT}^{16}$ on persons with HIV suggested that lymphocyte proliferation function was augmented significantly at the 6-month followup visit after 10 weeks of TC exercise. One (1) $\mathrm{CCT}^{21}$ and 3 $\mathrm{RCSs}^{23,25,26}$ suggested that the number of lymphocytes, mainly $\mathrm{CD} 4^{+}$, and the ratio of $\mathrm{CD}^{+} / \mathrm{CD} 8^{+}$were significantly higher in TC groups, compared to control groups. One (1) $\mathrm{RCS}^{24}$ examined the number and percentage of B-lymphocytes and suggested that the number of ZC-rosetteforming cells (B-lymphocytes) was lower in a TC group at resting status but increased significantly after 20 minutes of exercise, compared to controls.

One (1) $\mathrm{RCT}^{17}$ indicated that number of NK cells increased significantly after 6 months of TC practice, but $1 \mathrm{RCS}^{23}$ suggested that the percentage of NK cells decreased significantly in a TC group after 25 minutes of exercise. One RCS ${ }^{22}$ examined the effect of TC on circulating myeloid dendritic cells (the potent antigen-presenting cells linking innate and adoptive immunity) and indicated a favorable effect of TC exercise.

Five (5) studies, including 2 RCTs and 3 CCTs, examined the concentration of Igs in peripheral blood. One (1) RCT ${ }^{15}$ and 3 CCTs $^{18,20,21}$ suggested favorable effects of TC exercise on $\operatorname{IgG}$ and $\operatorname{IgA}$, while another $\mathrm{RCT}^{12}$ only suggested a favorable effect on IgG. One (1) $\mathrm{RCT}^{11}$ examined concentrations of complements $(\mathrm{C} 3, \mathrm{C} 4)$, and the results indicated that concentrations of C3 and C4 increased significantly after 12 weeks of TC exercise. One (1) $\mathrm{CCT}^{20}$ also suggested a favorable effect of TC exercise on C3.

\section{Study quality}

Jadad scores for the included RCTs ranged from 1 to 4 , with a value of 3 or above only for 2 studies. Levels of evidence for the included studies were ranked as " $\mathrm{A}$ " for 3 studies, " $\mathrm{B}$ " for 12 studies, and " $\mathrm{C}$ " for 1 study.

\section{Discussion}

This review aimed to assess the efficacy and the effectiveness of TC exercise for treating infectious diseases. Following a comprehensive search of existing literature, it was found that clinical trials of TC in patients with infectious diseases were very limited. Only one study ${ }^{18}$ examined clinical symptoms relevant to infections among female students. The results indicated that the durations of URTIs shortened significantly after 6 months of TC exercise. Instead, many studies in the field examined the effectiveness of TC exercise on improvement of human immune function. Overall, this review demonstrated that the available evidence suggested favorable effects of TC exercise both for increasing effective components of the immune system and for improving immune function, as indicated by functional measures of immunity.

The risk of bias for the studies examined in this review was assessed, based on the descriptions of randomization, allocation concealment, blinding, and withdrawals. ${ }^{10}$ A high risk of bias might have existed in some of the included trials, which might have led to false-positive results. Of the 7 included RCTs, only $3^{13,14,16}$ described method of randomization and allocation concealment; 2 RCTs ${ }^{13,16}$ adopted assessor blinding, and $4 \mathrm{RCTs}^{13-16}$ reported details about dropouts and withdrawals and adopted intention-to-treat 


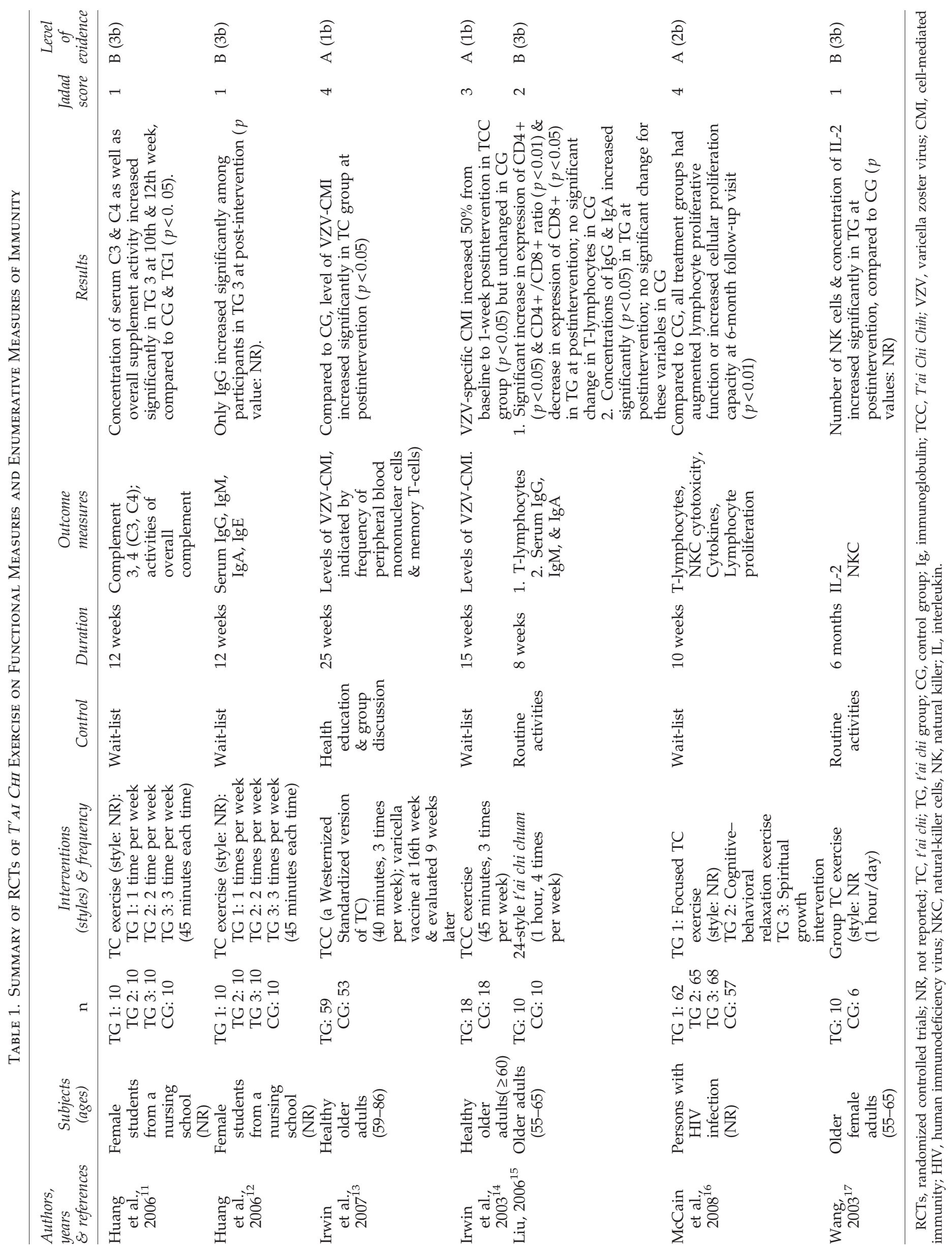




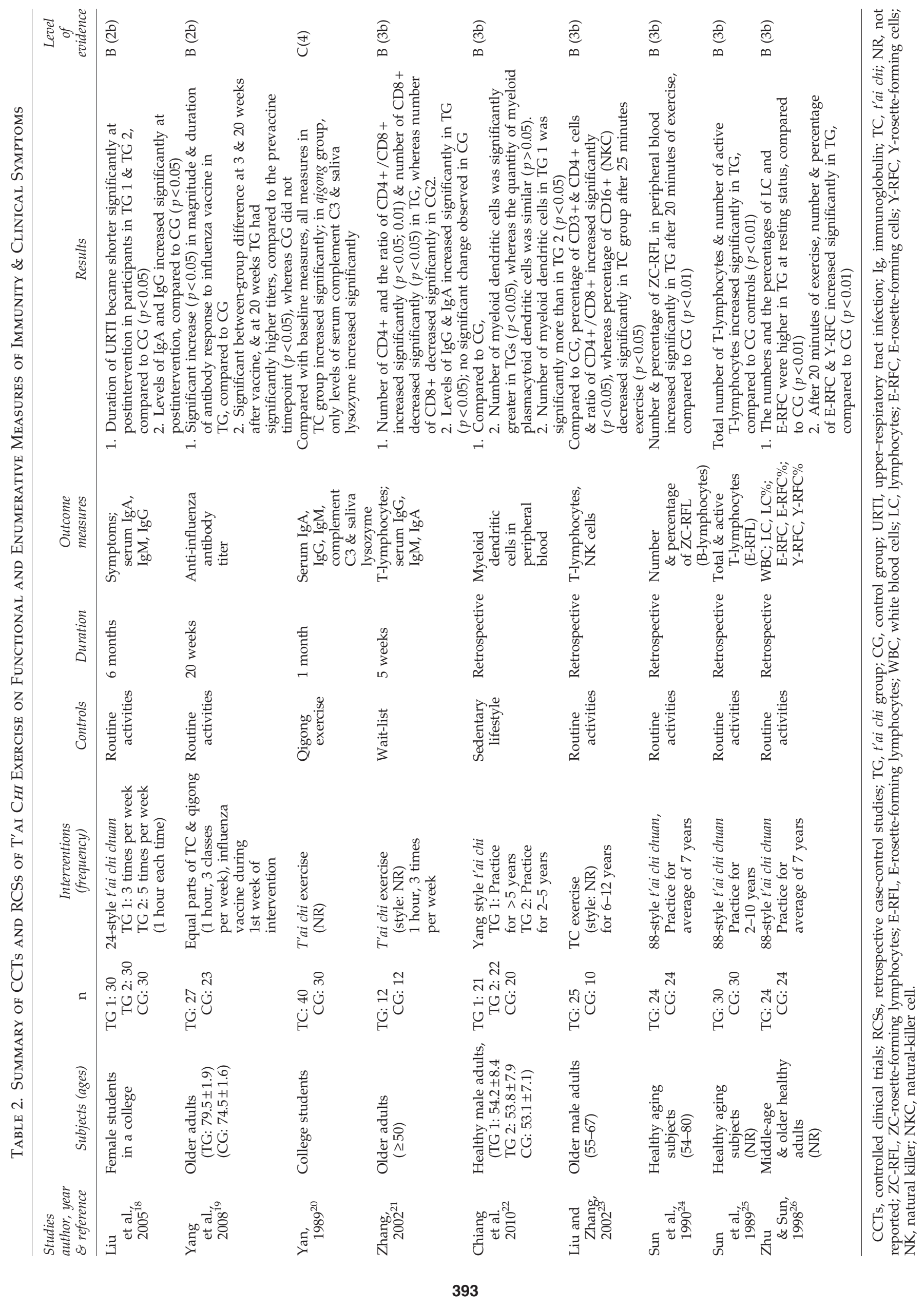


(ITT) analyses. The other RCTs did not have descriptions of their methods of sequence generation or allocation concealment and the details on dropouts, and were rated as "unclear" for these domains, thus, introducing the potential risk of bias. Details on dropouts and withdrawals were also described in $1 \mathrm{CCT}^{19}$ but the ITT analysis was not adopted in any CCT, which might have led to the exclusion of some particular patients and might have introduced attrition biases. The 4 included CCTs and 5 RCSs were subject to a high risk of selection bias caused by nonrandomized allocation. Moreover, the 5 included RCSs ${ }^{21-26}$ did not adjust the values of baseline measures; thus, the reliability of the evidence presented in these studies was clearly limited. One (1) study ${ }^{18}$ was presented at a conference on medical qigong and had not undergone the process of peer-review, thus, introducing potential for a number of biases. In the majority of the included prospective trials, group TC exercise training was provided preferentially to the intervention groups, whereas the control groups did not have a matched number of social contact hours with coparticipants. Thus, these studies might have been subject to potential risk of performance bias. Furthermore, the majority of the included studies had small samples (<50 subjects) and the results were prone to a type II error. Therefore, further vigorously designed, large-scale, placebo-controlled, randomized studies are needed.

Despite methodological flaws, nearly all of the included studies demonstrated a beneficial effect of TC exercise on one or more parameters of immunity. Apart from the included studies, one $\mathrm{CCT}^{27}$ of TC exercise on mediators (interleukins, transforming growth factor, and transcription factors) of the Th1/Th2/T regulatory reaction also suggested a beneficial effect of TC on improvement of T-cell helper function. All of the uncontrolled observational studies ${ }^{28-37}$ also reported favorable effects of TC exercise on different parameters of immunity, but such data were highly susceptible to bias and, hence, provided little scientific evidence of the specific effects of TC exercise for improving immune function.

It has been suggested that the mechanisms underlying exercise-associated immune changes are multifactorial and include many neuroendocrine factors. ${ }^{38,39}$ TC as a form of moderate-intensity exercise may promote release of neuroendocrinologic factors, such as catecholamines (adrenaline, noradrenaline), growth hormone, and cortisol, through the sympathetic-adrenal medullary (SAM) axis. ${ }^{39,40}$ These factors induce changes in cellular trafficking, lymphocyte proliferation, and antibody production. ${ }^{41}$ As a consequence of muscular contraction and catecholamine-induced immediate changes, for instance, cellular components of the immune system may be mobilized to the blood. ${ }^{38}$ Moreover, TC exercise may lead to an increased oxygen supply and alterations in metabolism and metabolic factors, such as plasma glutamine and plasma glucose, which also contribute to exercise-associated changes in immune function. ${ }^{38,39}$ An additional possibility is that immune enhancement is mediated, in part, by improvements of psychosocial factors that are promoted by TC as a mind-body intervention. ${ }^{42}$ It has been suggested that psychologic stress can affect immunity through the hypothalamic-pituitary-adrenal (HPA) axis. ${ }^{43}$ Stress-induced activation of the HPA axis results in the release of neuroendocrine hormones, such as adrenocorticotropin, from the anterior pituitary gland. Adrenocotropin then circulates through the bloodstream to the adrenal glands, where the hormone induces release of glucocorticoids, which can bind receptors at the cell surfaces of lymphoid and myeloid cells. ${ }^{44}$ TC as a form of mind-body intervention may buffer the effects of stress on plasma glucocorticoids and, thus, induce alterations in immune function.

It should be noted that the immune system is a complex system, and both functional and enumerative measures of immunity provide rough estimates of specific processes rather than global indications of the immune system's capacity to resist infectious disease. ${ }^{7}$ First, the normal functioning range is very broad for most immune measures, and it is still unclear whether or not the magnitude of changes induced by exercise are sufficient to move outside of the normal ranges. Even if these changes were sufficient, it is not clear whether or not the alterations would persist for a sufficient duration of time to alter risk for infectious disease. ${ }^{7}$ Second, most of the included studies were conducted using healthy adults, and the clinical implications of the changes in these parameters in healthy people are unclear. Changes in cell number may just reflect changes in the dynamics of lymphocyte migration and recirculation, or shifts in plasma volume, rather than absolute changes in total cell numbers. ${ }^{45}$ In addition, absolute changes in cell number will not necessarily result in a significant change in the capacity of the immune system to make an effective response to antigenic challenge. ${ }^{46}$ Thus, it would be inappropriate to conclude that TCinduced changes in any specific immune parameter signal a state of "immune enhancement" or resistance to infections. ${ }^{7}$

This review may have had some limitations. Similar to any systematic review, one major limitation was the potential incompleteness of the evidence reviewed. The aim was to identify all controlled trials in this area in a large number of databases with no restrictions on publication language. The current authors are confident that the search strategy used for this review had located all relevant data; however, a degree of uncertainty remains. Moreover, selective publishing and reporting can be major causes of bias in the included studies. In addition, it was not possible to perform metaanalyses because of the heterogeneity of study designs and outcome measures in the included studies.

\section{Conclusions}

The available evidence suggest that TC exercise may improve both cell-mediated immunity and antibody response in immune system, but it remains debatable whether or not the exercise-induced alterations in immune function are sufficient to alter human body defense, disease susceptibility, and severity. ${ }^{47}$ Because of methodological flaws in existing studies, further vigorously designed large-scale placebocontrolled, randomized trials are needed. Future studies should also test the efficacy of TC exercise for reducing the incidence or the severity of infectious disease.

\section{Acknowledgments}

This review was supported by the Hospital Authority of Hong Kong (HA105/48 PT5).

\section{Disclosure Statement}

No competing financial interests exist. 


\section{References}

1. Hall A, Maher C, Latimer J, Ferreira M. The effectiveness of tai chi for chronic musculoskeletal pain conditions: A systematic review and meta-analysis. Arthritis Rheum 2009;61:717-724.

2. Wang WC, Zhang AL, Rasmussen B, et al. The effect of tai chi on psychosocial well-being: A systematic review of randomized controlled trials. J Acupunct Meridian Stud 2009;2:171-181.

3. Yeh GY, Wang C, Wayne PM, Phillips R. Tai chi exercise for patients with cardiovascular conditions and risk factors: A systematic review. J Cardiopulm Rehabil Prev 2009;29:152160.

4. Irwin M, Pike J, Oxman M. Shingles immunity and health functioning in the elderly: Tai chi chih as a behavioral treatment. Evid-Based Complement Alternat Med 2004;1:223-232.

5. Nieman DC. Exercise and resistance to infection. Can J Physiol Pharmacol 1998;76:573-580.

6. Sim YJ, Yu S, Yoon KJ, et al. Chronic exercise reduces illness severity, decreases viral load, and results in greater antiinflammatory effects than acute exercise during influenza infection. J Infect Dis 2009;200:1434-1442.

7. Miller GE, Cohen S. Psychological interventions and the immune system: A meta-analytic review and critique. Health Psychol 2001;20:47-63.

8. Oxford Centre for Evidence-based Medicine. Levels of Evidence. March 2009. Online document at: www.cebm.net/ index.aspx?o=1025. Accessed November 10, 2010.

9. Jadad AR, Moore RA, Carroll D, et al. Assessing the quality of reports of randomized clinical trials: Is blinding necessary? Control Clin Trials 1996;17:1-12.

10. Jüni P, Altman DG, Egger M. Systematic reviews in health care: Assessing the quality of controlled clinical trials. BMJ 2001;323:42-46.

11. Huang QP, Jiang GF, Wan YP, Xiong ML. The effect of Taijiquan on enhancing overall supplement activity in serum in female college students [in Chinese]. J Phys Educ 2006;13:69-71.

12. Huang QP, Wan YP, Dai KX, Jiang GF, Tang SY. Human immune response of senior university female students enhanced by Taijiquan exercise [in Chinese]. J Wuhan Institute Phys Educ 2006;40:54-56.

13. Irwin MR, Olmstead R, Oxman MN. Augmenting immune responses to varicella zoster virus in older adults: A randomized, controlled trial of tai chi. J Am Geriatr Soc 2007;55:511-517.

14. Irwin MR, Pike JL, Cole JC, Oxman MN. Effects of a behavioral intervention, tai chi chih, on varicella-zoster virus specific immunity and health functioning in older adults. Psychosom Med 2003;65:824-830.

15. Liu XD. Effect of 8-week tai chi on immune function of older people [in Chinese]. Chin J Clin Rehabil 2006;10:10-12.

16. McCain NL, Gray DP, Elswick RK, et al. A randomized clinical trial of alternative stress management interventions in persons with HIV infection. J Consult Clin Psychol 2008;76:431-441.

17. Wang XJ. Effect of Taijiquan on IL-2 of aged people [in Chinese]. J Shandong Institute Phys Educ 2003;19:48-50.

18. Liu YH, Nie H, Ma GD, Zhou JQ, Su CL. Research on impact of Taijiquan on immunoglobulins and the relationship between immunoglobulins and Up-respiratory tract infections (URTI) in the female college students [in Chinese]. J Beijing Sport Univ 2005;28:1089-1090.
19. Yang Y, Verkuilen J, Rosengren KS, et al. Effects of a traditional taiji/qigong curriculum on older adults' immune response to influenza vaccine. Med Sport Sci 2008;52:64-76.

20. Yan H. Research on the relationship between immunity of human body and physical training, taijiquan and qigong. 2nd International Conference on Qigong, September 10-15, 1989, Xi'an, China.

21. Zhang $\mathrm{M}$. The impact of tai chi exercise on immune function in older adults [in Chinese]. J Henan Normal Univ (Natural Sci) 2002;30:85-88.

22. Chiang J, Chen YY, Akiko T, et al. Tai chi chuan increases circulating myeloid dendritic cells. Immunol Invest 2010;39:863-873.

23. Liu SH, Zhang $\mathrm{H}$. The research on the effect of tai chi exercise on T-lymphocyte subgroup and NK cells [in Chinese]. China Sport Sci Technol 2002;38:50-52.

24. Sun XS, Xu Y, Zhu R, Detection of ZC rosette-forming lymphocytes in the healthy aged with Taichiquan. J Sports Med Phys Fitness 1990;30:401-405.

25. Sun XS, Xu Y, Xia YJ. Determination of E-rosette-forming lymphocytes in aged subjects with Taichiquan exercise. Int J Sports Med 1989;10:217-219.

26. Zhu Y, Sun X. Effect of Taichiquan exercise on immunofunction in the aged [in Chinese]. J Shanghai Teachers Univ 1998;27:70-74.

27. Yeh SH, Chuang H, Lin LW. Regular tai chi chuan exercise improves T-cell helper function of patients with type 2 diabetes mellitus with an increase in T-bet transcription factor and IL-12 production. Br J Sports Med 2009;43: 845-850.

28. Yang KD, Chang WC, Chuang H, et al. Increased complement factor $\mathrm{H}$ with decreased factor $\mathrm{B}$ determined by proteomic differential displays as a biomarker of tai chi chuan exercise. Clin Chem 2010;56:127-131.

29. Lee EO, Chae YR, Song R, et al. Feasibility and effects of a tai chi self-help education program for Korean gastric cancer survivors. Oncol Nurs Forum 2010;37:E1-E6.

30. Yeh SH, Chuang H, Lin LW, Hsiao CY, Eng HL. Regular tai chi chuan exercise enhances functional mobility and CD4CD25 regulatory T-cells. Br J Sports Med 2006;40:239-243.

31. Jin $\mathrm{H}$. The impact of tai chi exercise on T-lymphocyte subsets in human peripheral blood [in Chinese]. Zhong Guo Yi Yue Xue Bao 2000;15:16-17.

32. Liu J, Chan XF, Chan PJ. Effect of long-term tai chi exercise on the concentration of serum IGF-I among middle-aged and older women [in Chinese]. Chin J Sports Med 2004;23: 686, 697 .

33. Liu J, Chan XF, Qiu PX. Effect of long-term tai chi exercise on NK T-cell counts among middle-aged and older women [in Chinese]. Chin J Sports Med 2007;26:738-739.

34. Liu J, Chan PJ, Wang Y. Effect of tai chi exercise on the concentrations of IFN-r and IL-4 in prepheral blood of middle-aged and older women [in Chinese]. Chin J Sports Med 2009;28:557-558.

35. Li ZQ, Sun X. Effect of Wu-style tai chi on NK cell activities in peripheral blood of older adults [in Chinese]. Chin J Sport Med 1995;14:53-56.

36. Qi DY, Li XH, Wang YG, Liu WH. A study of influence of Taijiquan exercise on immune function of diabetes II patients [in Chinese]. J Beijing Sport Univ 2008;31:932-933,950.

37. Esch T, Duckstein J, Welke J, Braun V. Mind/body techniques for physiological and psychological stress reduction: Stress management via tai chi training-a pilot study. Med Sci Monit 2007;13:CR488-CR497. 
38. Pedersen BK, Hoffman-Goetz L. Exercise and the immune system: Regulation, integration, and adaptation. Physiol Rev 2000; 80:1055-1081.

39. Pedersen BK, Toft AD. Effects of exercise on lymphocytes and cytokines. Br J Sports Med 2000;34:246-251.

40. Hoffman-Goetz L, Pedersen BK. Exercise and the immune system: A model of the stress response? Immunol Today 1994;15:382-387.

41. Goldrosen MH, Straus SE. Complementary and alternative medicine: Assessing the evidence for immunological benefits. Nat Rev Immunol 2004;4:912-921.

42. Kohut ML, Lee W, Martin A, et al. The exercise-induced enhancement of influenza immunity is mediated in part by improvements in psychosocial factors in older adults. Brain Behav Immun 2005;19:357-366.

43. Yang EV, Glaser R. Stress-associated immunomodulation and its implications for responses to vaccination. Expert Rev Vaccines 2002;1:453-459.

44. Eskandari F, Sternberg EM. Neural-immune interactions in health and disease. Ann N Y Acad Sci 2002;966:20-27.
45. Burns VE. Psychological stress and immune function. In: Gleeson M, ed. Immune Function in Sport and Exercise. Edinburgh \& New York: Churchill Livingstone Elsevier \& British Association of Sport and Exercise Sciences, 2006:221-246.

46. Vedhara K, Fox JD, Wang ECY. The measurement of stressrelated immune dysfunction in psychoneuroimmunology. Neurosci Biobehav Rev 1999;23:699-715.

47. Walsh NP, Gleeson M, Shephard RJ, et al. Position statement: Part one. Immune function and exercise. Exerc Immunol Rev 2011;17:6-63.

Address correspondence to: Chong-Wen Wang, MD, PhD

Center on Behavioral Health

The University of Hong Kong 5 Sassoon Road

Pokfulam, Hong Kong SAR

China

E-mail: wangcw@hku.hk 